\title{
Cylindrical-type nanometer-resolution laser diffractive optical encoder
}

\author{
Chi-Tang Hsieh and Chih-Kung Lee
}

\begin{abstract}
A new, to our knowledge, design for a cylindrical-type diffractive optical encoder is proposed. The wave-front aberrations induced by the power of the rotation disk in this encoder can be canceled out completely. Wave-front-aberration cancellation and desensitization to the grating misalignment are achieved by means of positioning the virtual point source, which was induced by the cylindrical grating with respect to two sets of modified telescopes with a magnification ratio of one: $1 \times$ telescopes. For evaluating the performance envelope of this newly designed optical system a CODE V-based optical-design software program was adopted to simulate the performance of the optical system. From toleranceanalysis results it was found that this newly developed cylindrical encoder system has the capability to compensate for most aberrations and, in addition, possesses a high tolerance for optical-component misalignment. For verifying the performance of the developed system the cylindrical diffractive encoder system was cross-referenced with a Hewlett-Packard Model HP-5529 laser interferometer positioning signal. The experimental results confirm the merits of this newly developed cylindrical encoder. (C) 1999 Optical Society of America
\end{abstract}

OCIS codes: $\quad 050.1940,090.1000,220.4830$.

\section{Introduction}

Classified by the shape of the grating used, rotaryand cylindrical-type diffractive optical encoders can both be used to measure the angular displacement of a moving object. Both types of diffractive optical encoder use a diffractive grating as the metric scale for measuring the displacement of an object with a nanometer-level resolution. The displacement of the diffractive grating relative to the encoder head modulates the phase of the diffracted light beams and generates interference fringes for the receiving optics to detect. Therefore a diffractive optical encoder ${ }^{1-8}$ is one of the most important of the key components for ultrahigh-precision positioning equipment such as mastering systems for optical storage systems or for servo track writers used in magnetic storage systems.

A rotary diffractive optical encoder ${ }^{9-11}$ uses a ra-

C.-T. Hsieh (hsieh@ahead.com.tw) is with AHEAD Optoelectronics, Inc., No. 13 Chin-ho Road, Chung-ho, Taipei hsien 235, Taiwan. C.-K. Lee (cklee@mems.iam.ntu.edu.tw) is with the Institute of Applied Mechanics, National Taiwan University, Taipei, Taiwan.

Received 1 February 1999; revised manuscript received 23 April 1999.

0003-6935/99/224743-08\$15.00/0

(C) 1999 Optical Society of America dial grating as the metric scale. As the grating pitch of a radial grating varies linearly along the radial direction, an incoming beam with a finite spot size will experience strong wave-front-aberration effects within the region of the diffracted light beams. These wave-front aberrations can present major difficulties in creating feasible interferometric configurations that are needed for high-performance laser encoders. Various optical designs have been proposed to minimize and compensate for radialgrating-induced wave-front aberrations. Review and analysis of these designs have prompted the development of a new cylindrical-type diffractive optical encoder.

In this study the optical components of this new, to our knowledge, design for an optical encoder system are arranged such that the wave-front aberrations induced by the power of the rotation disk can be canceled out completely. Furthermore, the system's performance degradation, which is due to misalignment between the moving grating and the rest of the encoder head, which is stationary, is drastically reduced. Wave-front-aberration cancellation and desensitization to the grating misalignment are achieved by means of positioning a virtual point source (VPS), which was induced by the power of the rotating disk on which a grating is located, with respect to two sets of modified telescopes with a magnification ratio of one: $1 \times$ telescopes. $^{12} \quad$ Each of the 


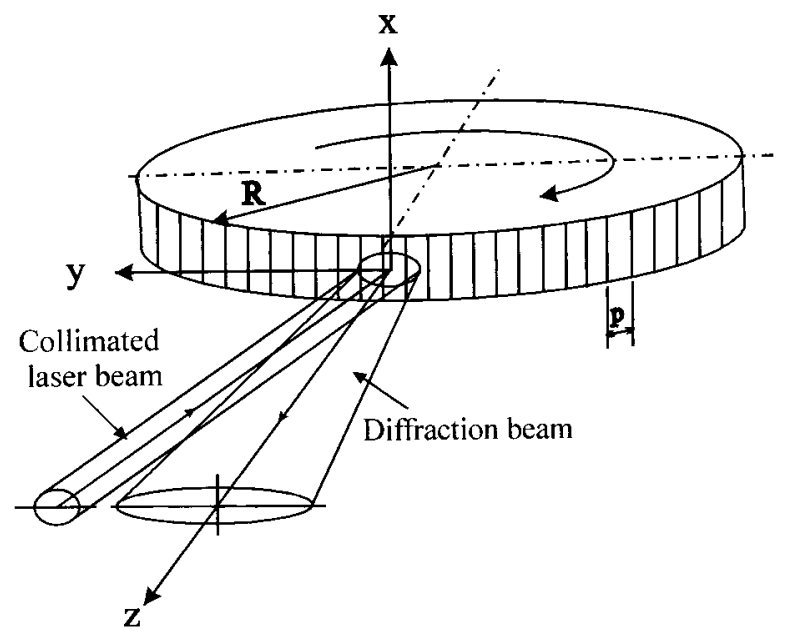

Fig. 1. Wave-front aberration induced by a rotating disk.

$1 \times$ telescopes comprises a spherical lens, a rightangle prism, and a rotation disk with a grating. This feature makes the installation of the encoder and the grating much easier because of the larger system-misalignment tolerance available. In addition, it provides the system with the capability to generate high-quality output signals even when the structures being measured are rotated at high speed and the vibrations of the grating and the structure add significant noise to the signals as a result of the misalignment.

To evaluate the performance envelope of the newly design optical system, we adopted CODE v-based ${ }^{13}$ optical design software to simulate the performance of the optical system. From the tolerance-analysis results it was found that this newly developed cylindrical encoder system has the capability to compensate for most aberrations, and, in addition, possesses a high tolerance for optical-component misalignment. To verify the performance of the developed system, we cross-referenced the cylindrical diffractive encoder, which was constructed by use of discrete components placed on top of a conventional optical table, with a Hewlett-Packard Model HP-5529 laser interferometer ${ }^{14}$ positioning signal. The experimental results confirm the merits of the newly developed cylindrical encoder and offer proof of its highprecision position-detection capabilities, its high optical-component misalignment tolerance, and its excellent wave-front-aberration immunity.

\section{Cylindrical-Type Diffractive Optical Encoder}

Cylindrical-type diffractive optical encoders ${ }^{15,16}$ use linearly shaped diffraction gratings mounted on the circumference of the rotation disk as the metric scale. The rotation of the diffraction grating relative to the encoder head modulates the phase of the diffracted beam and generates an interference fringe so the receiving optics can produce quadrature signals and deduce the angular position of the rotation disk. When collimated laser beams impinge on the grating

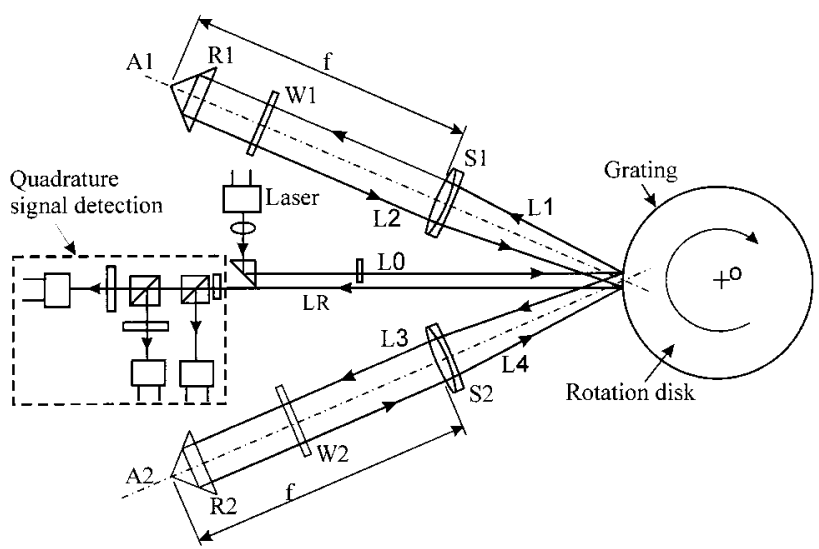

(a)

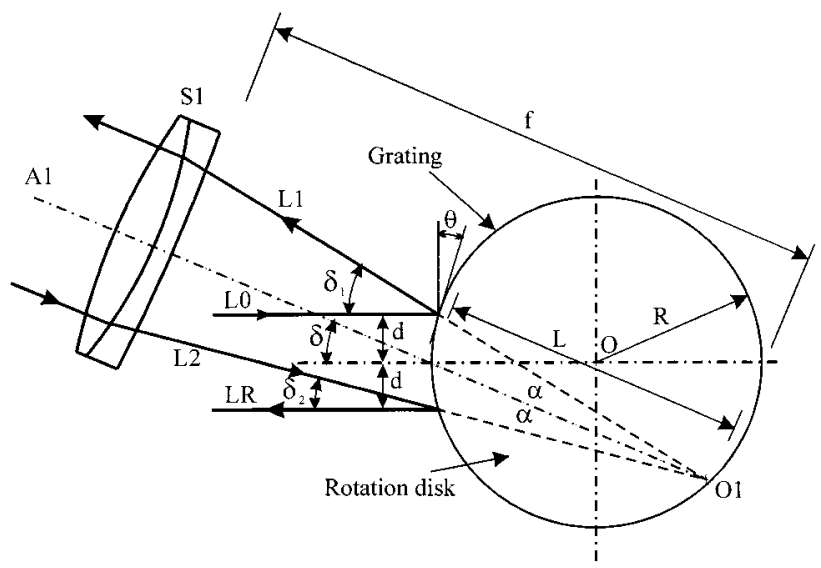

(b)

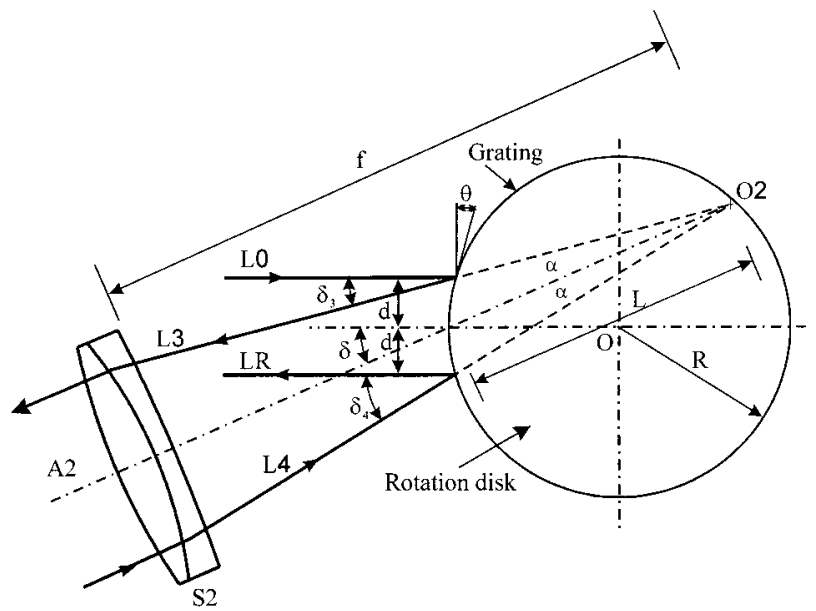

(c)

Fig. 2. (a) New design for a cylindrical-type diffractive optical encoder. Close-up views of a cylindrical diffractive optical encoder system for (b) a +1 -order diffracted beam and (c) a -1-order diffracted beam.

the diffracted beams will suffer wave-front aberration, such as astigmatism, that is induced by the power of the rotation disk on which the diffraction grating sits (Fig. 1). The diffracted-beam wavefront aberration induced by the power of the rotation disk will degrade the interference fringe's signal-to- 


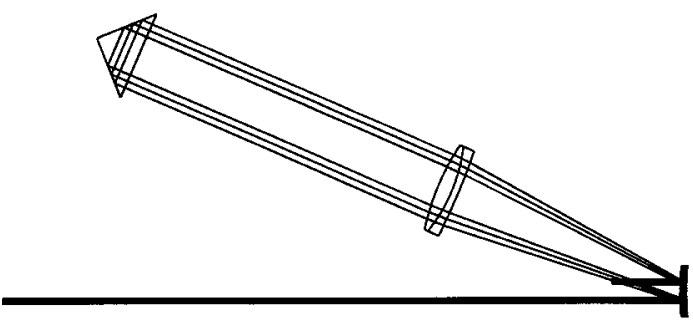

$35.71 \mathrm{~mm}$

Fig. 3. Diagram of the optical simulation for a +1 -order diffracted beam in which the designed grating pitch is $1.6 \mu \mathrm{m}$, the wavelength is $632.8 \mathrm{~nm}$, and the radius of the rotation disk is $110 \mathrm{~mm}$.

noise ratio and also make system performance sensitive to optical-component misalignment.

For compensating the wave-front aberration the new cylindrical-type diffractive encoder's optical design, shown in Fig. 2(a), uses two sets of modified $1 \times$ telescopes to locate the VPS. The collimated laser beam then impinges on the reflective diffraction grating and diffracts along the +1 -order and the -1 -order diffraction beams along the $L 1$ and the $L 3$ directions, respectively. From Fig. 2(b), we can see that the resultant +1-order diffraction beam is diffracted along the direction of the angle $\delta_{1}$, which can then be calculated from the grating equation and the geometric relation, as given by

$$
\delta_{1}=\left[\sin ^{-1}\left(\frac{\lambda}{p}+\frac{d}{R}\right)+\sin ^{-1}\left(\frac{d}{R}\right)\right],
$$

where $\lambda$ is the wavelength of the measuring laser, $p$ is the pitch of the reflective diffraction grating, $d$ is the offset distance between the incidence point and the center point, and $R$ is the radius of the rotational disk. The optical system is arranged such that the nominal center line of a spherical lens $S 1$, the oneeighth-wave plate $W 1$, the right-angle reflector $R 1$, and the optical axis $A 1$ are all aligned along the direction of the angle $\delta$, given by the following condition:

$$
\delta=\frac{1}{2}\left[\sin ^{-1}\left(\frac{\lambda}{p}+\frac{d}{R}\right)+\sin ^{-1}\left(\frac{\lambda}{p}-\frac{d}{R}\right)\right] .
$$

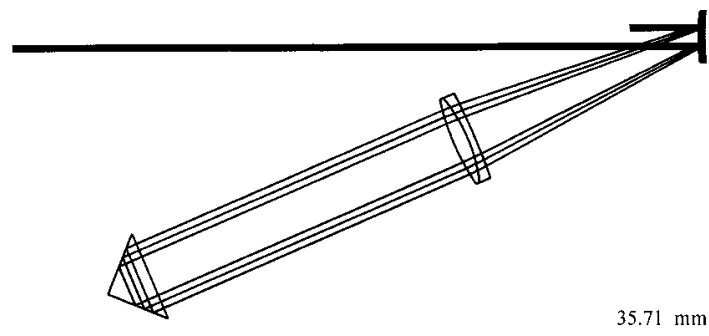

Fig. 4. Diagram of the optical simulation for a-1-order diffracted beam in which the designed grating pitch is $1.6 \mu \mathrm{m}$, the wavelength is $632.8 \mathrm{~nm}$, and the radius of the rotation disk is $110 \mathrm{~mm}$.

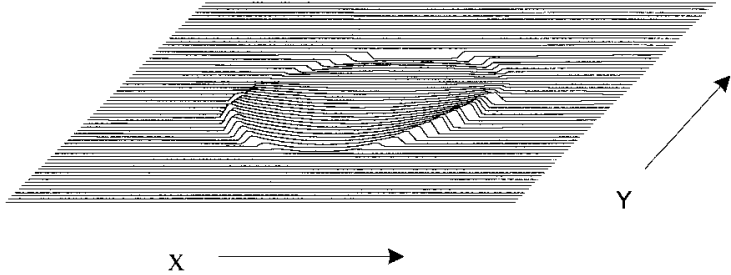

Fig. 5. Quality of the rms wave front of a +1 -order returning beam for which the rms and the peak-to-valley wave-front errors are 0.005 and 0.025 wave, respectively.

The spherical lens is located at a distance $f$ from $\mathrm{VPS}_{O 1}$, and the right-angle reflector is also located at a distance $f$ from the spherical lens, where $f$ is the focal length of the spherical lens. The roof edges of right-angle reflectors $R 1$ and $R 2$ are oriented parallel to the grating line of the diffraction grating. Furthermore, $\mathrm{VPS}_{O 1}$ is located along the optical axis at a distance $L$, as measured from the central point and given by

$$
\begin{aligned}
L= & \frac{\sin (\pi / 2-\delta)}{\sin \alpha}[d+R(1-\cos \theta) \tan \delta] \\
& +R(1-\cos \theta) \sec \delta,
\end{aligned}
$$

where $\alpha=1 / 2\left[\sin ^{-1}(\lambda / p+d / R)-\sin ^{-1}(\lambda / p-d / R)\right]$ $+\sin ^{-1}(d / R)$ and $\theta=\sin ^{-1}(d / R)$. The spherical lens, the right-angle prism, and the rotation disk with the grating together make up the $1 \times$ modified telescope. In this structure the +1 -order diffracted beam passes through the spherical lens, is collimated, then passes through the one-eighth-wave plate, and is reflected back by the right-angle reflector. The reflected light beam then passes again through the one-eighth-wave plate and the spherical lens. The resultant beam is then focused toward VPS $_{O 1}$ in the direction of angle $\delta_{2}$, which is given by

$$
\delta_{2}=\left[\sin ^{-1}\left(\frac{\lambda}{p}-\frac{d}{R}\right)-\sin ^{-1}\left(\frac{d}{R}\right)\right]
$$

and is incident upon the same reflective diffraction grating. Thus the rediffracted beam is again collimated when its propagation direction moves in the direction of $L R$ toward the signal detector in the opposite direction of the original incident beam $L 0$ [Fig. $2(\mathrm{a})]$.

Likewise, as can be seen from Fig. 2(c), the -1order diffraction beam diffracts from the reflective

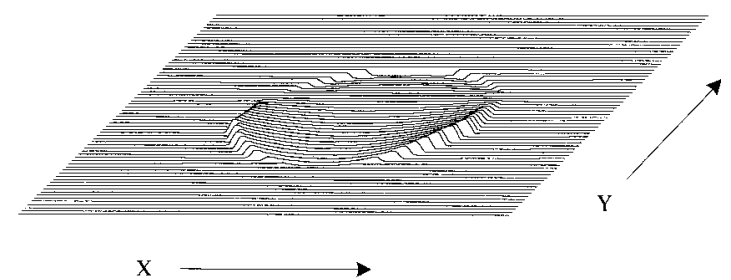

Fig. 6. Quality of the rms wave front of a -1-order returning beam for which the rms and the peak-to-valley wave-front errors are 0.005 and 0.025 wave, respectively. 

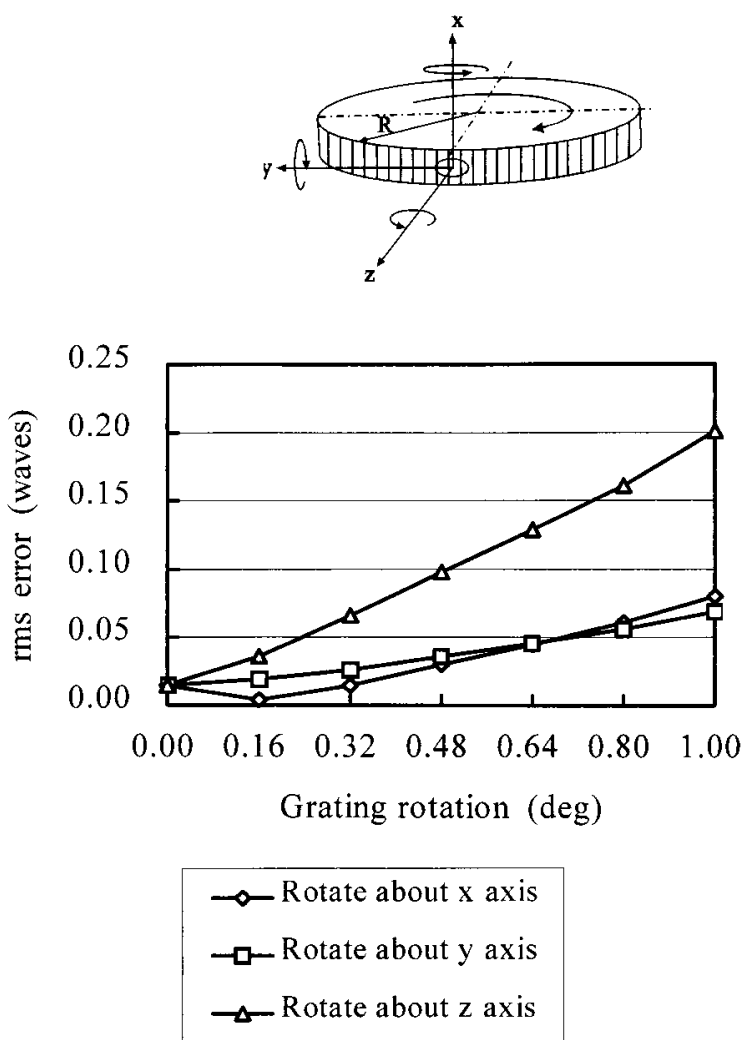

Fig. 7. Quality of the rms wave front versus the disturbance in the grating rotation.

diffraction grating along $L 3$ in the direction of angle $\delta_{3}$ and can be given by

$$
\delta_{3}=\left[\sin ^{-1}\left(\frac{\lambda}{p}-\frac{d}{R}\right)-\sin ^{-1}\left(\frac{d}{R}\right)\right] .
$$

The optical system is arranged so that the nominal center line of the spherical lens $S 2$, the one-eighthwave plate $W 2$, and the right-angle reflector $R 2$ are disposed along the optical axis $A 2$ and aligned in the direction of the angle $\delta$, where

$$
\delta=\frac{1}{2}\left[\sin ^{-1}\left(\frac{\lambda}{p}+\frac{d}{R}\right)+\sin ^{-1}\left(\frac{\lambda}{p}-\frac{d}{R}\right)\right] .
$$

The spherical lens is located at a distance $f$ from $\mathrm{VPS}_{O 2}$, and the right-angle reflector is also located at the distance $f$ from the spherical lens, where $f$ is the focal length of the spherical lens. Additionally, $\mathrm{VPS}_{\mathrm{O} 2}$ is located along the optical axis at a distance $L$ from the central point, as given by

$$
\begin{aligned}
L= & \frac{\sin (\pi / 2-\delta)}{\sin \alpha}[d+R(1-\cos \theta) \tan \delta] \\
& +R(1-\cos \theta) \sec \delta .
\end{aligned}
$$

The -1-order diffraction beam passes through the collimating spherical lens, then passes through the one-eighth-wave plate, and is reflected back by the right-angle reflector to pass through the one-eighth-
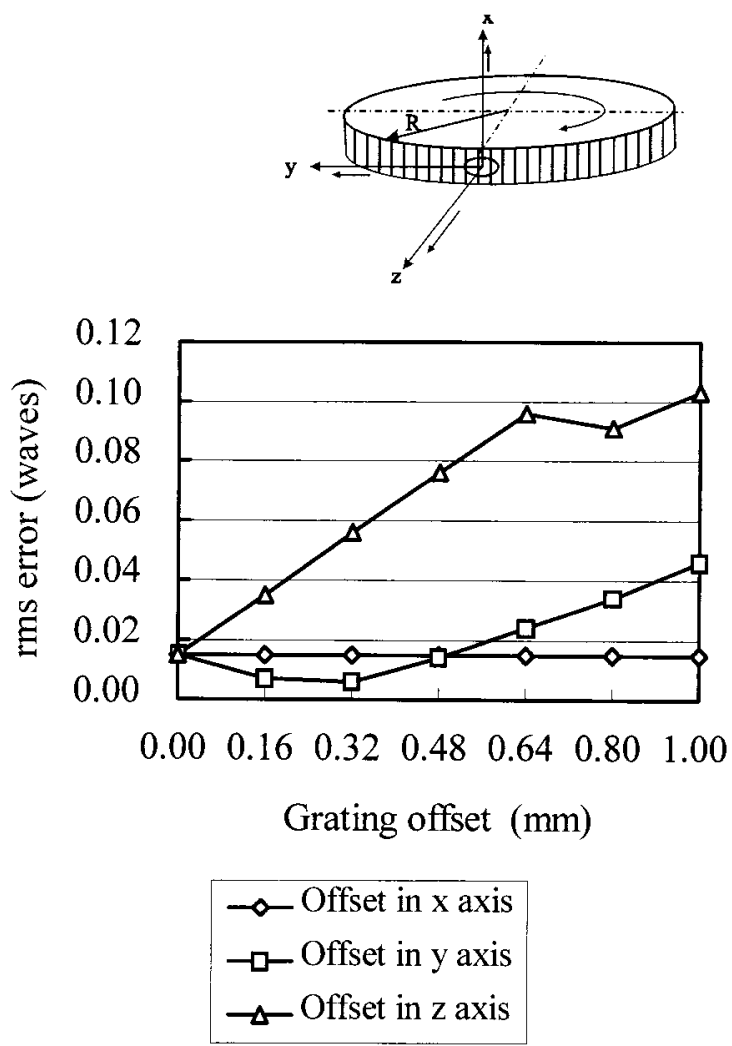

Fig. 8. Quality of the rms wave front versus the disturbance in the grating offset.

wave plate and the spherical lens. The resultant beam is then focused toward VPS $\mathrm{O}_{2}$ in the direction of angle $\delta_{4}$, as given by

$$
\delta_{4}=\left[\sin ^{-1}\left(\frac{\lambda}{p}+\frac{d}{R}\right)+\sin ^{-1}\left(\frac{d}{R}\right)\right],
$$

and is incident upon the reflective diffraction grating. The rediffracted beam is again collimated and coincides with the +1 -order return beam in the direction $L R$ directed toward the signal detector.

In this newly designed system the wave-front aberrations caused by the power of the rotation disk on the diffracted light beams are Fourier transformed ${ }^{17}$ by the modified $1 \times$ telescopes' optical layout. The returning beams are again Fourier transformed and diffracted a second time by the same grating. Because the light beams diffracted from the grating have a wave front that is antisymmetric about the central point of the reflective diffraction grating, the returning light has a wave front that is complex conjugated to the preceding light beams that were diffracted from the same grating. Therefore, the +1 -order and the -1 -order returning light along the $L R$ direction will be collimated again after the second diffraction from the same grating. With the optical layout presented in this newly developed encoder the wave-front aberrations induced by the power of the rotation disk can be canceled out completely. Furthermore, the system can tolerate a much larger 

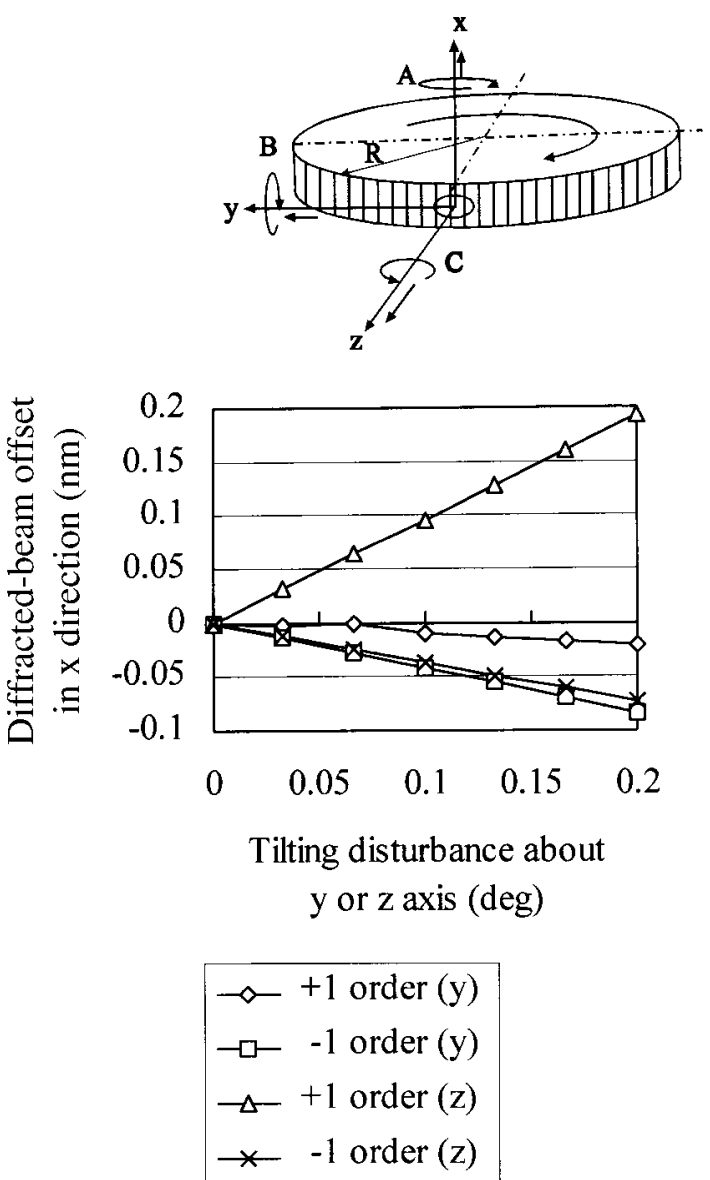

Fig. 9. Offset of the diffracted beam in the $x$ direction that was induced by a tilting disturbance about the $y$ or the $z$ axis. The offset of the diffracted beam is negligible in the $x$ direction for cases of offset disturbance along the $x$, the $y$, and the $z$ directions and for cases of tilt about the $x$ axis.

range of misalignment of the optical components and, as a result, can tolerate much larger mounting errors of the diffraction grating.

\section{Optical Simulation}

CODE v-based optical design software was adopted for modeling the optical system and evaluating the performance envelope of the newly design optical system. In our model the radius of the rotation disk and the pitch of the linear sinusoidal grating were attached to the circumference of the rotation disk at $110 \mathrm{~mm}$ and $1.6 \mu \mathrm{m}$, respectively. From Eq. (2) the proper layout of the spherical lens and the rightangle prism can be calculated. The distances from the spherical-lens front surface to $\mathrm{VPS}_{O 1}$ and $\mathrm{VPS}_{O 2}$ are given in Eqs. (3) and (7).

Figures 3 and 4 show the optical design model of the +1 -order and the -1 -order diffraction beams, respectively, after optimization of the optical system. The rms wave-front quality of the +1-order and the -1 -order returning beams is shown in Figs. 5 and 6 . To examine the compensating capability of the design system, we added some disturbance to the rotation disk. Figures 7 and 8 reveal the rms wave-front
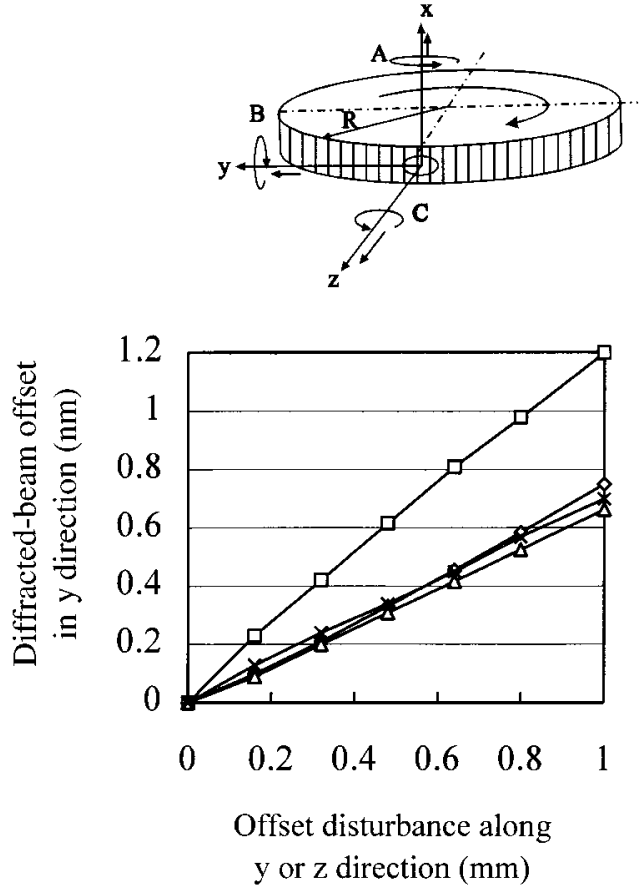

(a)

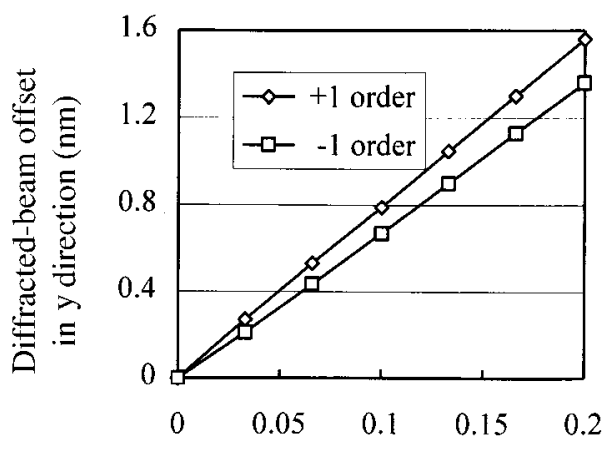

Tilting disturbance about $\mathrm{x}$ axis (deg)

(b)

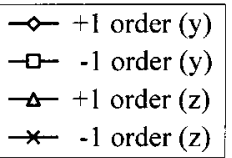

Fig. 10. Offset of the diffracted beam in the $y$ direction (a) as induced by an offset disturbance along the $y$ or the $z$ direction and (b) as induced by a tilt disturbance about the $x$ axis. Note that the offset of the diffracted beam in the $y$ direction is negligible for cases of an offset disturbance along the $x$ direction and for cases of tilt disturbance about the $y$ or the $z$ axis.

quality of the designed optical system versus the displacement and the rotation disturbance of the rotation disk. From the simulation results it can be seen that the system is quite robust to misalignment of the rotation disk with respect to the encoder head. Because the diffractive optical encoder uses the +1 order and the -1-order output diffracted beams to 

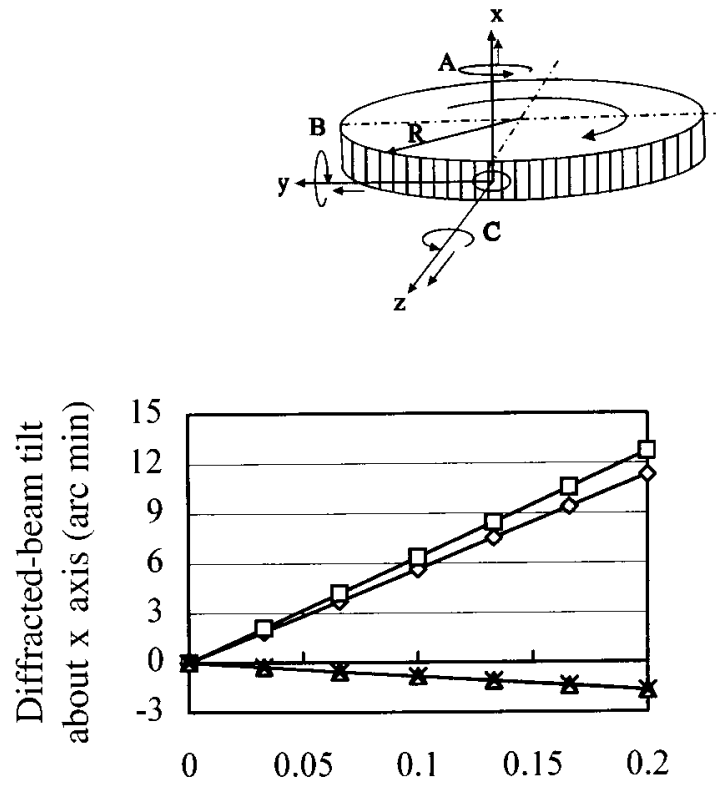

Tilting disturbance about y or $z$ axis (deg)

$$
\left[\begin{array}{ll}
\neg- & +1 \text { order }(\mathrm{y}) \\
-\square- & -1 \operatorname{order}(\mathrm{y}) \\
-\Delta-1 & +1 \operatorname{order}(\mathrm{z}) \\
-\mathrm{x} & -1 \text { order }(\mathrm{z})
\end{array}\right]
$$

Fig. 11. Tilt of the diffracted beam about the $x$ axis that is induced by the tilt disturbance about the $y$ or the $z$ axis. The tilt of the diffracted beam about the $x$ axis is negligible for cases of offset disturbance along the $x$, the $y$, or the $z$ direction and for cases of tilting about the $x$ axis.

obtain interference fringes for detecting angular displacement, it is very important to know the relative orientations of the +1 -order and the -1 -order diffracted beams at the output end. Figures 9-12 show the relative orientations of the +1 -order and the -1-order returning beams as measured at a distance of $200 \mathrm{~mm}$ from the grating after the disturbance of the rotation disk described above. From the tolerance-analysis results it was found that this newly developed cylindrical encoder system has the capability to compensate for most aberrations as well as maintain a high tolerance to optical-component misalignment.

\section{Experimental Results}

Figure 13 shows a schematic of the experimental setup. A $1.6-\mu \mathrm{m}$-pitch reflective holographic grating was mounted on the circumference of the rotation disk, which had a radius of $110 \mathrm{~mm}$. A He-Ne gas laser with a wavelength of $632.8 \mathrm{~nm}$ was used as the measuring light source. After proper alignment of the optical system, as described in Section 3, the quadrature signal was obtained by the signal detector (Fig. 14). To verify the performance of the system, we mounted a reflector from the Model HP-5529
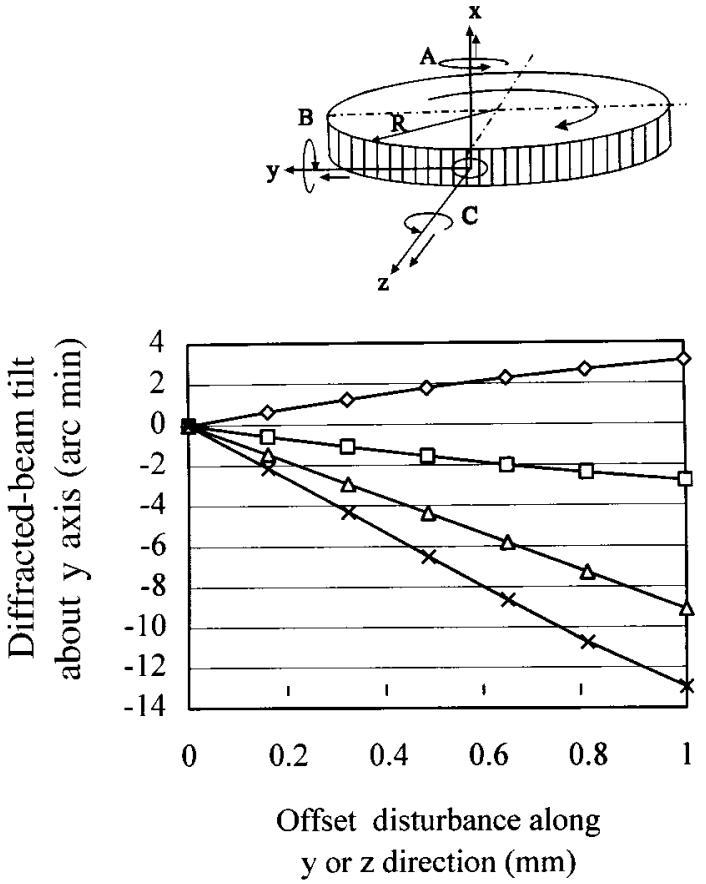

(a)

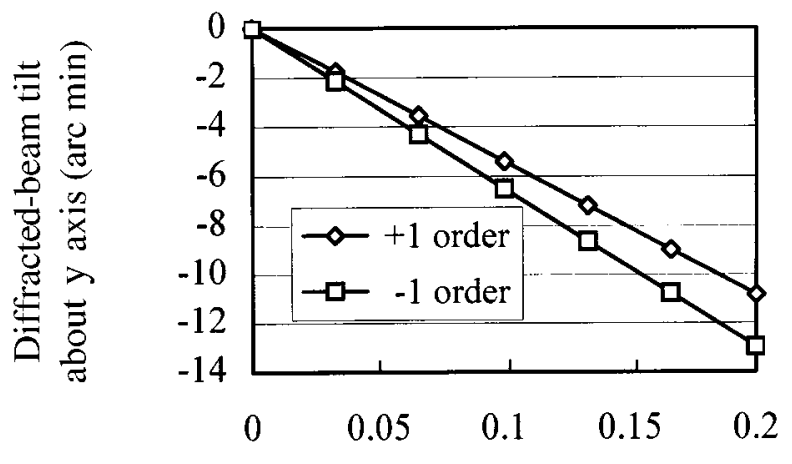

Tilting disturbance about $\mathrm{x}$ axis ( $\mathrm{deg})$

(b)

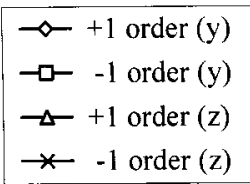

Fig. 12. Tilt of the diffracted beam about the $y$ axis (a) as induced by an offset disturbance along the $y$ or the $z$ direction and (b) as induced by tilt disturbance about the $x$ axis. Note that the tilt of the diffracted beam about the $y$ axis is negligible for cases of offset disturbance along the $x$ direction and for cases of tilt about the $y$ or the $z$ axis.

interferometer on top of the rotation disk and aligned it with the edge of the grating. When the disk rotates at a small angle with respect to its center, the encoder system and the Model HP-5529 interferometer, both of which are controlled by their own separate PC systems running in a Microsoft Windows environment, take the displacement data with an ex- 


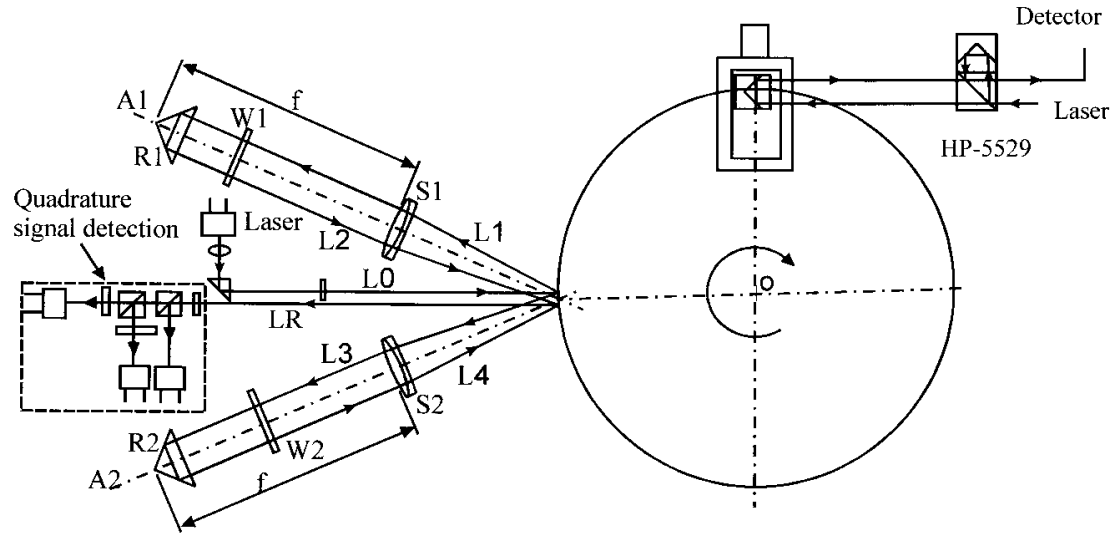

Fig. 13. Diagram of the experimental setup of the cylindrical diffractive optical encoder.

ternal trigger signal. Because both controlling PC systems execute their sampling programs under an event-driven system such as Microsoft Windows, the sampling-action start times of the two PC systems do not coincide exactly because the PC's operating systems dictate the start of the sampling action. This timing discrepancy appears as a horizontal shift be-

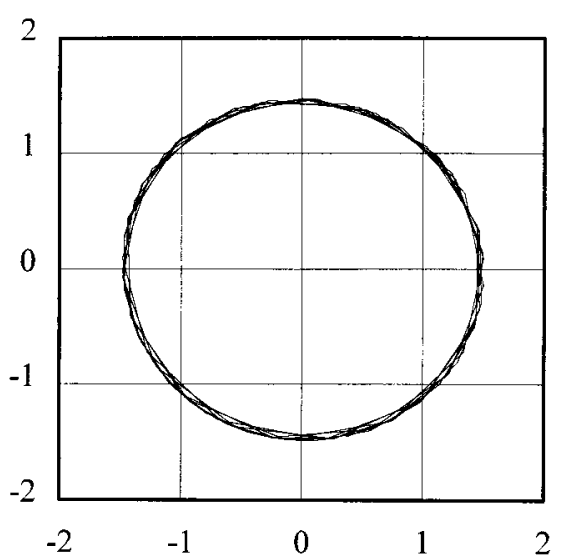

Fig. 14. Quadrature signals detected by the signal detector.

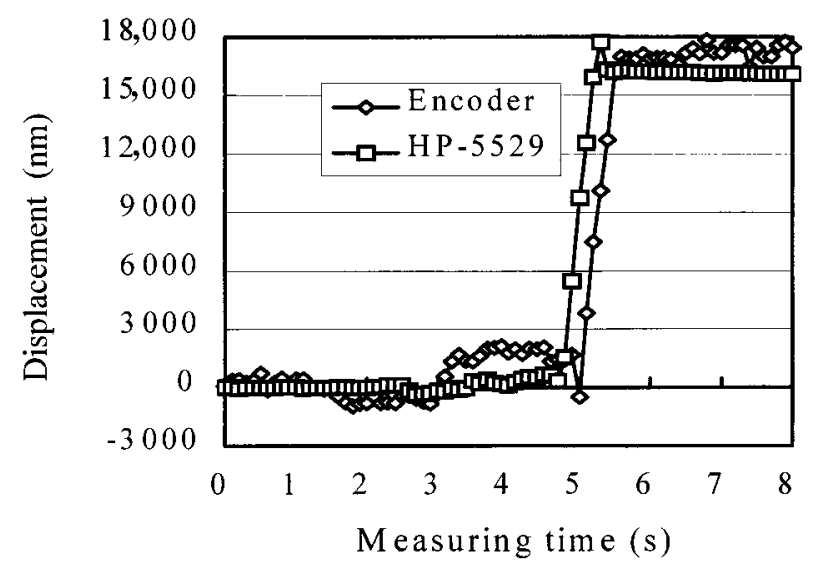

Fig. 15. Experimental results for two sets of data: the proposed cylindrical diffractive optical encoder and the Hewlett-Packard Model HP-5529 laser interferometer. tween the two sets of data shown in Fig. 15. In a geometrical sense, the encoder system output and the Model HP-5529 interferometer should take the same value. The experimental data shown in Fig. 15 indicate that the maximum discrepancy in vibration induced by disk rotation between the two systems is less than $5 \mu \mathrm{m}$. With all the optical components still mounted on top of a regular optical table and not reduced into a compact package, the system is not immune to external vibration, noise, air-flow fluctuations, and so on. The 5- $\mu \mathrm{m}$ error obtained appears to be satisfactory even though more-detailed experiments are currently underway.

\section{Conclusions}

A newly designed optical system with a high tolerance for misalignment between the moving grating and the rest of the encoder head, which is stationary, has been presented. Wave-front-aberration cancellation and desensitization to grating misalignment are achieved by means of positioning the VPS, which was induced by the cylindrical grating with respect to two sets of a modified $1 \times$ telescopic optical layout. From tolerance-analysis results it has been proven that this newly developed cylindrical encoder system has the capability to compensate for most aberrations and, in addition, possesses a high tolerance to opticalcomponent misalignment. The performance of the designed system was cross-referenced with a Model HP-5529 laser interferometer positioning signal. The experimental results have confirmed the merits of the newly developed cylindrical encoder and have proved its high-precision position-detection capabilities, high optical-component misalignment tolerance, and excellent wave-front-aberration immunity.

\section{References}

1. T. Nishimura and K. Ishizuka, "Laser rotary encoder," Motion Sept./Oct., 14 (1986).

2. K. Ishizuka, T. Nishimura, and O. Kasshara, "Encoder," UK patent GB 2,185,314 (15 July 1987).

3. K. Ishizuka and N. Kawasaki, "Encoder with high resolving power and accuracy," U.S. patent 5,146,085 (8 September 1992). 
4. D. K. Mitchell and G. Thorburn, "Apparatus for detecting relative movement wherein a detecting means is positioned in the region of natural interference," U.S. patent 5,486,923 (23 January 1996).

5. S. Ishii, T. Nishimura, K. Ishizuka, and M. Tsukiji, "Optical type encoder including diffraction grating for producing interference fringes that are processed to measure displacement," U.S. patent 4,912,320 (30 July 1990).

6. O. P. Lausanne, "Diffraction photoelectric displacement measuring device," U.S. patent 4,938,595 (3 July 1990).

7. S. Ichikawa and H. O. Kawasaki, "Diffraction-type optical encoder with improved detection signal insensitivity to optical grating gap variations," U.S. patent 4,943,716 (24 July 1993).

8. K. Ishizuka, T. Nishimura, and O. Kasahara, "Rotary encoder using reflected light," U.S. patent 5,036,192 (30 July 1991).

9. W. W. Chiang and C. K. Lee, "Wavefront reconstruction optics for use in disk drive position measurement system," U.S. patent 5,442,172 (15 August 1995).
10. Laser Rotary Encoder, Catalog no. 0696SZ02 (Canon, Inc., Lake Success, New York, 1996).

11. B. Horwitz, "Diffractive technique to improve encoder performance," Laser Focus World Oct. 143-148 (1996).

12. K. Iizuka, Engineering Optics (Kyoristsu Shuppan, Tokyo, 1983).

13. CODE V User's Manual (Optical Research Associates, 3280 East Foothill Boulevard, Pasadena, Calif., 1996).

14. Laser and Optics Users Manual (Hewlett-Packard, 5651 West Manchester, Los Angeles, Calif., 1992).

15. M. Dobosz, "New style probe with interferometric transducer for surface roughness and form profiling," Opt. Eng. 33, 902907 (1994).

16. C. T. Hsieh, "Theoretical design and experimental implementation of cylindrical diffractive optical encoders," Ph.D. dissertation (National Taiwan University, Taipei, Taiwan, 1997).

17. J. W. Goodman, Introduction to Fourier Optics (McGraw-Hill, New York, 1968). 